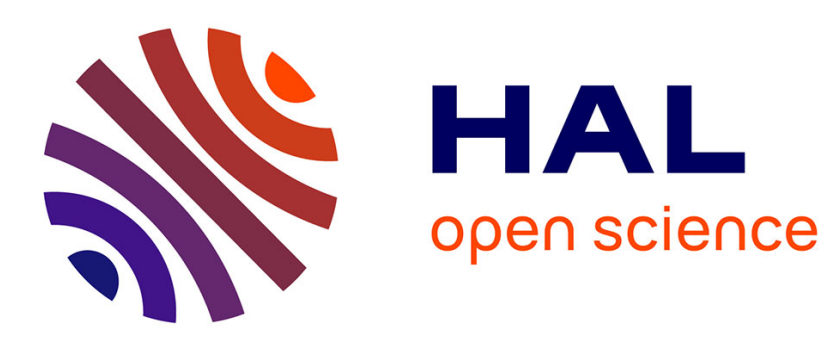

\title{
Anatomie animale: le festin carné des dieux d'après les textes hittites III.Le traitement des viandes
}

\author{
Alice Mouton
}

\section{To cite this version:}

Alice Mouton. Anatomie animale : le festin carné des dieux d'après les textes hittites III.Le traitement des viandes. 2007. halshs-00359771

\section{HAL Id: halshs-00359771 \\ https://shs.hal.science/halshs-00359771}

Preprint submitted on 9 Feb 2009

HAL is a multi-disciplinary open access archive for the deposit and dissemination of scientific research documents, whether they are published or not. The documents may come from teaching and research institutions in France or abroad, or from public or private research centers.
L'archive ouverte pluridisciplinaire HAL, est destinée au dépôt et à la diffusion de documents scientifiques de niveau recherche, publiés ou non, émanant des établissements d'enseignement et de recherche français ou étrangers, des laboratoires publics ou privés. 
Anatomie animale : le festin carné des dieux d'après les textes hittites III. Le traitement des viandes

Alice Mouton, CNRS Strasbourg

Dans les deux précédentes parties de cette étude ${ }^{1}$, les principales viandes offertes en sacrifice alimentaire aux dieux hittites ont été énumérées, ceci à la lumière des descriptions de rituels et de fêtes religieuses que nous connaissons. Il nous reste à considérer la ou plus exactement les manières dont sont traitées ces viandes lors du sacrifice. Ce troisième volet ne fera pas allusion aux différences culturelles qui semblent transparaître à travers les différents moyens de traiter la viande, cet aspect ayant été l'objet de ma contribution au VIe Congrès International d'Hittitologie ${ }^{2}$.

Première étape : la consécration de la victime sacrificielle (ou de la viande)

Il paraît logique de penser que la consécration de l'offrande alimentaire est une phase indispensable au processus du sacrifice sanglant. Le fait que les viandes sacrificielles (voire l'animal tout entier dont celles-ci sont extraites) doivent être consacrées est notamment illustré par les deux éléments suivants. Tout d'abord, cette consécration est manifeste dans la désignation même des viandes sacrificielles par le terme hittite šuppa «(viandes) sacrées/consacrées ». En second lieu, plusieurs textes hittites font allusion à la consécration préalable dont bénéficient les viandes. Dans le texte $n^{\circ} 8$ décrivant un rituel en l'honneur du dieu de l'orage de Kuliwišna, par exemple, le foie et le cœur sont qualifiés de šuppiššaranta « consacrés ».

Le processus de consécration des viandes sacrificielles regroupe deux actions essentielles : 1) la purification de la victime qui devient ainsi propre à la consommation divine ${ }^{3}$; 2) la dédicace de cette victime à la divinité concernée par la cérémonie.

1) Dans le texte $n^{\circ} 62$, on envisage de faire l'offrande au dieu de l'orage de Kuliwišna d'un bœuf ou d'un mouton. Or, le texte précise : "Mais le cuisinier consacre (l'éventuel) bœuf (ou) mouton avec du tuhhueššar. " Le tuhhueššar est une substance fréquemment employée dans les rituels sous forme d'une fumigation ${ }^{4}$. Son rôle est généralement purificateur. De même, il ne me paraît pas impossible que le texte $n^{\circ} 64$ fasse également allusion au rituel de consécration de la victime avant qu'elle ne soit abattue. En effet, ce texte indique : «On [a]mène un taureau (var. une vache). Il (var. le prêtre) place du bois de cèdre (sur) sa tête et ses épaules. » où le possessif renvoie très vraisemblablement à l'animal. Le bois de cèdre est bien connu dans les textes religieux hittites (et même plus généralement proche-orientaux) pour ses vertus purificatrices lorsqu'il est employé en fumigation. Il semble que la purification de la victime sacrificielle soit l'étape la plus importante dans le processus de consécration de cette dernière. B. J. Collins (Collins 1995: 79 note 6) cite en effet le passage du rituel KUB 25.20 + KUB 46.23 iv 15'-16' dans lequel on fait allusion aux paroles de consécration des animaux destinés à être sacrifiés, paroles qui sont prononcées par le chef des cuisiniers (UGULA ${ }^{\text {LÚ.MEŚ }}$ MUHALDIM šupp [iyahh]uwaš INIM ${ }^{\mathrm{HA}}$ memai). Or ces paroles

\footnotetext{
${ }^{1}$ Ces deux parties ont été publiées dans Colloquium Anatolicum 3 (2004): 67-92 et Colloquium Anatolicum 4 (2005): 139-154.

${ }^{2}$ Cette contribution est intitulée « Quelques différences régionales concernant le sacrifice sanglant en Anatolie hittite $»$.

${ }^{3}$ Voir Mouton 2004: 311-312.

${ }^{4}$ Mouton, à paraître.
} 
font exclusivement référence à l'état de pureté que doivent atteindre l'autel, les victimes sacrificielles $^{5}$, le pain ordinaire et le vase à libation.

2) A. Goetze (Goetze 1970: 88) suggère que le terme šipant- soit parfois employé pour désigner l'acte de dédicace de la victime à la divinité. Sa proposition se justifie par le fait que, dans plusieurs textes $\left(\mathrm{n}^{\circ} 12,24,32\right.$, etc.), l'animal est toujours vivant après avoir été traité par l'action šipant- (Goetze 1970: $88 \mathrm{n}^{\circ} 145$ ). Je pense toutefois que la traduction traditionnelle « sacrifier» reste valide, puisque ce verbe implique avant tout le geste de la donation d'une victime animale à la divinité et, seulement consécutivement à cette offrande, à l'immolation de l'animal. La personne qui dédie la victime sacrificielle à la divinité est généralement un prêtre ou le commanditaire du rituel, selon la circonstance $\left(e x: n^{\circ} 62\right)$. Dans le texte $n^{\circ} 62$, on peut penser que le geste de la dédicace de la victime sacrificielle, geste parfois rendu par le verbe šipant-, est décrit précisément comme suit: "On les [=les victimes sacrificielles] amène, le maître de maison s'agenouille derrière eux et dit en face du dieu en personne : 'Je viens de (te) vouer celui-ci et celui-là pour cette affaire. Maintenant, je viens de les apporter à la divinité. [Vois]-les devant (toi), divinité !'» Il s'agit donc, pour le dédicant, de s'agenouiller derrière les victimes et de les " présenter » à la divinité en tant qu'offrandes lui étant destinées. Bien sûr, il ne s'agit là que d'une des multiples manières dont les Hittites devaient offrir leurs offrandes alimentaires à leurs dieux.

Seule la combinaison de ces actions 1) et 2) doit faire de l'animal et plus précisément des morceaux de viandes qui en sont extraits des šuppa, c'est-à-dire des viandes consacrées. Une fois les viandes offertes au dieu, elles deviennent sacrées, comme tout ce qui touche de près la divinité. Reste à tenter de déterminer si l'on pouvait consacrer directement les viandes que l'on souhaitait offrir au dieu ou bien s'il était nécessaire de consacrer l'animal tout entier.

Quoi qu'il en soit, il faut noter que ce premier geste dédicatoire consistant à consacrer l'animal ou des parties de son corps ne représente généralement que la première étape du processus du sacrifice. L'animal qui a été présenté et voué à la divinité afin d'être consacré doit par la suite être abattu. De son corps sont extraites les viandes qui constitueront le repas divin. Il arrive même que l'on présente d'abord ces dernières crues à la divinité puis qu'on les retire de devant le dieu pour les cuire. Ce n'est qu'après leur cuisson que ces viandes sont remises à leur emplacement initial (texte $\left.n^{\circ} 4\right)$.

\section{Deuxième étape : l'abattage de l'animal}

L'étude des verbes employés pour décrire l'abattage des victimes sacrificielles a déjà été faite ${ }^{6}$. Ces verbes sont principalement : 1) $h a t t(a)$ - ; 2) $k u(e) n$ - et 3) $h u(e) k$-.

1) hatt(a)- est généralement traduit par «piquer, frapper, percer, atteindre (à l'aide d'une flèche) » voire «marquer $»^{7}$. Il a généralement pour objet direct l'animal lui-même $\left(n^{\circ} 14,26\right)$ ou le pronom personnel qui le représente $\left(n^{\circ} 8,12,37\right.$, etc.). A. Goetze traduit hatt(a)- par «cut open » (Goetze 1970: 90) alors que C. Kühne (Kühne 1986: 93 et note 33 pour bibliographie) propose la traduction plus spécifique de « (die Kehle/die Halsschlagadern)

\footnotetext{
${ }^{5}$ Dans ce contexte, c'est plus précisément l'auli- des victimes qui doit être pur, ce qui indique que cet organe est un élément central lors de l'abattage de l'animal. Ce fait est d'ailleurs corroboré par les textes nº62 et 63 . Dans le texte $\mathrm{n}^{\circ} 62$, plus particulièrement, il est clair que l'auli- est un endroit par lequel on peut vider l'animal de son sang, d'où la traduction « jugular veins » employée par plusieurs auteurs dont le plus récemment B. J. Collins (Collins 1995: 79 note 6).

${ }^{6}$ Goetze 1970 ; Ünal 1985: 431-432 ; Collins 1995.

${ }^{7}$ HED III: 248-254. Pour le sens de « marquer », en particulier lorsque le verbe apparaît sous la forme hazziya-, voir Mouton 2004a: 100.
} 
durchschneiden oder -stechen ", traduction que reprennent le CHD (CHD L-N: 188) et B. J. Collins (Collins 1995: 80 : "slit the throat»). Cette seconde interprétation du verbe, bien qu'extrapôlant sans doute quelque peu le terme hittite, ne doit pas être très éloignée de la vérité. Le texte $n^{\circ} 9$ nous indique en effet : "Quand il/elle sacrifie un agneau, il/elle le tue sur la berge (d'une rivière) (namma-an-kan wappui katta hattai) et laisse le sang (couler) par terre. »L'action décrite par le verbe hatt(a)- implique donc un écoulement du sang de l'animal, ce qui va bien dans le sens d'un égorgement.

2) $k u(e) n$ - « tuer » est également employé, bien que beaucoup plus rarement, pour faire allusion à l'abattage d'une victime sacrificielle $\left(n^{\circ} 36\right)$. Le verbe est bien connu et n'a probablement pas une acception technique particulière dans ce contexte.

3) $h u(e) k$ - est généralement traduit par «tuer, abattre ». Contrairement à $k u(e) n-$, il semble réservé à l'abattage des animaux et ne peut pas être employé en rapport avec un être humain. Ce verbe n'est pas très souvent utilisé dans le contexte du sacrifice sanglant. On le retrouve dans les textes $n^{\circ} 27,30,57$ et 62 par exemple.

A. Goetze (Goetze 1970: $88 \mathrm{n}^{\circ} 150$ et 151) a remarqué la pratique, rarement attestée dans nos textes, consistant à assommer la victime sacrificielle avant de l'abattre. Le verbe employé pour désigner cette action est walh- «frapper». L'extrait $\mathrm{n}^{\circ} 151$ de Goetze indique que ce geste peut être effectué sur un bœuf, et ce au moyen d'un "bâton en fer » ( $t$-an AN.BAR-a

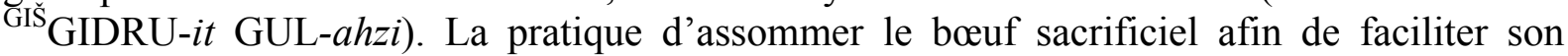
abattage (le plus souvent par égorgement) n'est pas une chose inattendue : il n'est pas évident de parvenir à égorger un bovidé qui se débat de toutes ses forces ! L'extrait n 150 de Goetze nous apprend que cette pratique était également utilisée de temps à autres pour les ovidés. Cette fois, c'est un objet lithique qui sert à assommer l'animal, geste exécuté, dans ce

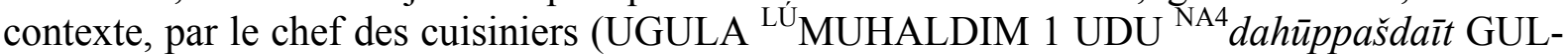
$a h z i)$.

L'abattage est souvent ritualisé ${ }^{8}$ et se pratique assez fréquemment dans un lieu sacré ou du moins consacré. Les textes $n^{\circ} 27, n^{\circ} 30$ et $n^{\circ} 57$ indiquent en effet que l'animal peut être abattu en face d'une stèle huwaši. Lorsque le lieu n'est pas sacré en lui-même, il arrive qu'on le consacre par un rituel impliquant l'utilisation de tuhhueššar, comme le remarquait très justement B. J. Collins (Collins 1995: 79). D'autres textes nous apprennent toutefois que l'abattage peut s'effectuer dans un lieu apparemment dénué de toute signification symbolique ou religieuse. On peut notamment mentionner la «maison du cuisinier » (É ${ }^{L U}$ MUHALDIM : $\left.n^{\circ} 8\right)$.

L'identité des personnes qui sont chargées de procéder à l'abattage de l'animal n'est presque jamais spécifiée. Les textes désignent généralement l'auteur ou les auteurs de cette action par la troisième personne du pluriel qui équivaut au pronom indéfini français « on ». Parmi les extraits qui sont étudiés dans cette série d'articles, seul le texte $\mathrm{n}^{\circ} 9$ utilise la troisième personne du singulier dans ce contexte (namma-an-kan wappui katta hattai). Etant donné le caractère lacunaire du texte précédant l'extrait en question, il ne nous est malheureusement pas possible de déterminer l'identité du personnage ainsi désigné. Quoi qu'il en soit, le fait que les textes emploient le plus souvent l'indéfini indique peut-être que l'identité précise de la ou des personnes procédant à l'abattage proprement dit n'est pas un élément déterminant dans le rituel. Il me semble que cela tend également à montrer que ce n'est généralement ni le prêtre ni le commanditaire du rituel qui se charge de cette tâche particulière. Certains textes nous fournissent de plus amples informations sur ce point précis. C'est le cas des extraits $n^{\circ} 61$

\footnotetext{
${ }^{8}$ Sur d'autres aspects du rituel effectué autour de l'abattage de l'animal et en particulier sur le bruit/la musique accompagnant cet événement, voir Collins 1995: 82.
} 
et 62 qui indiquent qu'un cuisinier pouvait se charger de l'abattage ${ }^{9}$. Quant au texte $n^{\circ} 63$, il montre que cette tâche pouvait également incomber aux chefs des bergers fournisseurs des moutons de sacrifice. Enfin, KUB 53.14 ii 10-11 (qui correspond à un autre extrait du texte $\left.\mathrm{n}^{\circ} 4\right)$ indique que le gardien du temple pouvait également jouer ce rôle (1 UDU ${ }^{\mathrm{LU}} M A S A R$ É $\operatorname{DINGIR}^{L I M} \bar{e} p z[i] n-a n$ INA É DINGIR ${ }^{L I M}$ haššl $A N A{ }^{\mathrm{D}}$ telipinu huekzi « Le gardien du temple prend un mouton et il l'abat pour Telipinu dans un foyer dans le temple. »).

Il y a tout lieu de penser que le traitement du sang a une importance non négligeable lors du sacrifice $^{10}$. Plusieurs textes indiquent qu'il faut vider l'animal sacrificiel de son sang avant de pouvoir le déposer devant les dieux. Ce cas de figure semble se rapprocher quelque peu du modèle grec tel que J. - L. Durand le décrit ${ }^{11}$. Celui-ci précise que la manière la plus efficace de vider un animal de tout son sang est de lui trancher si ce n'est toute la trachée, du moins les deux carotides (Durand 1979: 137). On fait couler le sang de l'animal par terre $\left(n^{\circ} 9\right)$ ou dans un dépôt préalablement creusé (n³5). Dans KBo 29.65 iv 20' cité par C. Kühne (Kühne 1986: 95-96), le sang n'est pas gaspillé mais est au contraire recueilli dans un récipient ( $\mathrm{UDU}^{\top}-m a-(\check{s}) \check{s} a n$ ANA ${ }^{\mathrm{DUG}} \mathrm{LAHT}[\mathrm{A} \check{s} a r] \bar{a}$ hūganzi «On abat un mouton au-dessus d'un récipient LAHTA »). Il a alors un rôle dans le déroulement du rituel, même si le texte n'en dit pas plus à ce sujet. Le texte $\mathrm{n}^{\circ} 41$ indique que les victimes sacrificielles sont abattues audessus de pains ordinaires, ce qui pourrait signifier que l'on fait en sorte que leur sang soit absorbé par ces pains. Le texte $\mathrm{n}^{\circ} 34$ indique en outre que le sang d'un animal est parfois manipulé lors du rituel. Il peut même être parfois mélangé à un aliment lors de la préparation d'un pain de sacrifice $\left(\mathrm{n}^{\circ} 20\right)$. A côté du sang sorti du corps de la victime par égorgement, il arrive que l'animal sacrifié soit offert à la divinité « dans (son) sang » (ěšhani : texte n³6 où l'animal sacrifié est un porc) ; mais cela doit avoir lieu dans des contextes particuliers.

Observait-on le comportement de l'animal pendant que celui-ci était conduit au sacrifice ? B. J. Collins le pense mais se base, pour l'affirmer, sur les versions hittites du traité oraculaire šumma immeru $^{12}$. Ces textes ayant très bien pu servir de simples exercices scolaires, il me semble qu'on ne peut pas se fier à ces témoignages pour en déduire l'existence d'une telle pratique chez les Hittites. Le texte $\mathrm{n}^{\circ} 15$ me paraît un témoignage plus fiable. On y lit en effet : «Le commanditaire du rituel [place] la main sur le mouton. Si (c'est) son désir, il in[terroge] le foie, mais si (autre est) son désir, il n'[interroge] pas le foie. » Il arrive donc que le foie de l'animal sacrifié soit interrogé au cours d'une cérémonie, et cela n'est, à mon sens, pas surprenant outre mesure. Il paraît en effet logique de penser que l'on exploite pleinement un animal sacrifié (qui est par essence coûteux). On profiterait donc de l'occasion du sacrifice pour inspecter les entrailles de la victime afin de répondre à l'une des questions qui préoccupent le commanditaire du rituel (le plus souvent le roi ${ }^{13}$ ) à ce moment-là. Car le commanditaire du rituel est le donateur de l'animal et le revendique souvent en plaçant sa

\footnotetext{
${ }^{9}$ Contrairement à ce que semble penser Mazoyer 2002: 85, aucun élément ne permet de supposer l'existence de « prêtres-bouchers ». Les textes font seulement allusion à de simples cuisiniers. Ceux-ci font sans doute partie du personnel du temple de la divinité honorée par le sacrifice.

${ }^{10}$ Sur l'importance du sang dans la religion hittite en général, voir Beckman, à paraître. Je remercie le Pr. G. Beckman de m'avoir transmis cet article.

${ }^{11}$ Durand 1979: 137 : «Autour de la blessure, une certaine quantité de sang subsiste, retenue dans les chairs et les tissus lésés. Il reste à la libérer dans un système de contraintes où le sang est situé hors du consommable parce que réservé au divin. »

${ }^{12}$ Collins 1995: 81 : « In addition, a recent study of Hittite 'šumma immeru' texts has brought to light some evidence that the Hittites may have observed the victim's behavior as it was led to slaughter and examined its entrails afterwards. » Voir également Hoffner 1993: 116-119.

${ }^{13} \mathrm{C}$ 'est en effet le plus souvent le roi qui est au centre des rituels magiques ainsi que des fêtes religieuses étudiés ici.
} 
main sur ce dernier (voir par exemple texte $\mathrm{n}^{\circ} 15$ ). Il est par conséquent directement concerné par l'interrogation oraculaire pratiquée à cette occasion. Il serait d'autant plus logique que l'on utilise les entrailles de l'animal sacrificiel pour la divination que cet animal et en particulier ses membres ont été apprêtés rituellement pour devenir porteurs d'une certaine sacralité, ce qui est propice, me semble-t-il, à véhiculer un message divin ${ }^{14}$. Cette interprétation pourrait en outre expliquer en partie la présence fréquente de devins lors des fêtes religieuses. Il faut par ailleurs souligner que l'examen des entrailles d'un animal présuppose de toute évidence -et ceci malgré le mutisme des textes hittites à ce sujet ${ }^{15}$ - le sacrifice en bonne et dûe forme de celui-ci. Ce phénomène est bien attesté pour la Syrie ancienne notamment ${ }^{16}$, et il doit, à mon avis, être également une réalité de l'Anatolie hittite.

Il semble donc que l'animal sacrificiel soit généralement égorgé avant d'être offert à la divinité. Quelques textes font toutefois référence à l'immolation par le feu de la victime entière, mais sans doute s'agit-il de contextes bien particuliers. On citera le cas du texte $\mathrm{n}^{\circ} 18$ qui décrit un rituel d'évocation dans lequel il est dit que l'agneau destiné au sacrifice est entièrement brûlé. De même, le texte $\mathrm{n}^{\circ} 65$ fait allusion à la combustion complète des šuppa dans le cadre d'une offrande ambǎšsi $i$.

\section{Troisième étape : le débitage de la viande}

Deux principaux verbes sont généralement considérés comme pouvant se rapporter à la phase du débitage de la victime sacrificielle : 1) ark- et 2) mark-.

1) Le verbe ark- semble avoir toujours pour objet direct l'animal $\left(n^{\circ} 5,23,44,45\right)$ ou le pronom personnel qui le représente $\left(n^{\circ} 24,38\right)$, à l'instar de $\operatorname{hatt}(a)-{ }^{17}$. Dans un rituel pour les déesses-mères et les Gulšeš $\left(\mathrm{n}^{\circ} 9\right)$ ark- succède à hatt- . Ce sont les principales raisons pour lesquelles la plupart des hittitologues ayant étudié ce verbe ont préféré le traduire par « diviser, découper » et donc " débiter» dans le contexte qui nous concerne (HED I: 140-142 et $\mathrm{HW}^{2} \mathrm{~A}:$ 300-301). D'après J. Puhvel, il pourrait également s'agir de tracer ou marquer le corps (de l'animal) en portions (HED I: 141) ${ }^{18}$. L'interprétation du CHD (CHD L-N: $188^{19}$ ) et celle de B. J. Collins (Collins 1995: 80), à savoir que le verbe ark-désigne l'action

\footnotetext{
${ }^{14} \mathrm{~J}$. -J. Glassner m'a rappelé à juste titre que, dans la divination mésopotamienne, les exta ne sont porteuses d'oracles qu'à partir du moment de l'abattage de l'animal. Pour une idée analogue au sujet de la divination étrusque, voir Briquel 2004: 152-153 : « La dimension divinatoire du sacrifice tyrrhénien, à travers l'examen des exta, est donc la réponse des dieux dans l'acte sacrificiel, ce qu'ils donnent à l'homme en retour. C'est pourquoi on ne doit pas considérer comme absurdes les théories, que raille Cicéron dans son traité sur la divination, voulant que les signes n'existassent pas dans les organes des victimes avant le sacrifice : ils pouvaient assurément être compris comme n'apparaissant qu'au moment du sacrifice, et résulter de cet échange entre hommes et dieux, comme réponse des dieux à l'acte d'offrande dont ils bénéficiaient de la part des hommes. C'était là la contrepartie des dieux dans l'échange. »

${ }^{15}$ Concernant ce silence des sources hittites, B. J. Collins (Collins 1995: 80) a sans doute raison d'écrire : «Perhaps we should assume that this is an omission of the obvious since numerous Boghazköy texts record the results of extispicy. »

${ }^{16}$ Lafont 1999: 62 : « Il est alors intéressant de relever, dans le dossier des lettres de devins, l'usage fréquent du verbe naqûm : celui-ci signifie clairement 'sacrifier', au sens de 'verser le sang', le substantif nîqum décrivant l'activité du devin. Il faut donc en conclure que la technique de l'examen des entrailles d'un animal est ici comprise comme un 'sacrifice'. Ce type de sacrifice à des fins oraculaires ne doit pas être mis à part des autres, et il faut au contraire considérer qu'aucun sacrifice n'était effectué à Mari sans intervention du devin. »

${ }^{17}$ Goetze 1970: $91 \mathrm{n}^{\circ} 171$ traduit notre extrait $n^{\circ} 24$ différemment de ce qui a été proposé dans le premier volet de cet article et en déduit que le verbe ark- peut avoir comme objet direct des parties du corps de l'animal. La traduction que j'ai quant à moi proposée s'oppose à cette interprétation.

${ }^{18}$ Idée reprise par Mazoyer 2002: 85.

19 « After the blood is drawn off, the carcass is skinned and gutted and some of the extremities removed (the verb ark- seems to cover this entire process). »
} 
d'écorcher et d'étriper l'animal, est judicieuse sans être vérifiable pour l'instant. Encore une fois, le contexte ne nous permet pas de savoir précisément de quel geste il s'agit. Les textes ne relatent que très rarement -voire peut-être jamais ?- l'ensemble des étapes jalonnant le processus d'abattage de la victime sacrificielle. Il se peut donc que le verbe ark- ne désigne qu'une seule des actions évoquées ci-dessus bien qu'il reste délicat de déterminer laquelle.

2) mark- est toujours placé après hatt(a)- et/ou ark- . Il a pour objet direct l'animal sacrifié $\left(n^{\circ} 23\right)$ et, plus rarement, des parties du corps de ce dernier $\left(\mathrm{n}^{\circ} 35\right.$ : «Puis on débite la 'main', les têtes (et) les 'pieds' »), ce qui valide la traduction " débiter » pour ce verbe. Les textes font parfois allusion au fait que l'animal entier est traité par l'action mark- $\left(\mathrm{n}^{\circ} 23\right)$ ou qu'il est mark- «nature » (pittalwant : $\mathrm{n}^{\circ} 9$ ) c'est-à-dire peut-être sans la peau et les entrailles (CHD L-N: 188), bien que cette interprétation ne soit pas assurée.

La principale difficulté qui découle de l'étude de ark- et de mark-consiste à trouver ce qui différencie ces deux verbes l'un de l'autre, puisqu'il a été suggéré qu'ils ont tous deux à faire avec la phase de débitage de la viande. A. Goetze, cherchant à marquer cette distinction, suggère de traduire ark- par « dissect» et mark- par " divide ». Cette traduction ne peut pas être rendue littéralement en français, où le verbe "disséquer» a le sens spécifique de " découper le corps d'un animal/d'un être humain dans le but de l'étudier ». Quoi qu'il en soit, il semble bien que les deux verbes hittites se rapportent à la notion de découpage du corps animal. Le fait qu'ils puissent se succéder dans un même passage indique cependant clairement que leur sens n'est pas entièrement redondant, d'où les nuances que l'on a cherché à attribuer à ark- .

A côté de ces deux verbes fréquemment employés dans les textes hittites, on constate l'utilisation beaucoup plus rare d'arha happešnai- dans le contexte du sacrifice sanglant. Ce verbe est, depuis l'étude d'A. Goetze (Goetze 1970: 91) traditionnellement traduit par « dépecer », " démembrer ». Cette interprétation se justifie principalement par le fait que le nom ${ }^{\mathrm{UZU}}$ happeššar / ${ }^{\mathrm{URU}}$ happešnant- désigne manifestement les différentes parties d'un corps, ce qui équivaut au sumérien ${ }^{U Z U} \mathrm{UR}^{20}$. La traduction anglaise d'A. Goetze « dismember » est donc celle qui semble se rapprocher le plus du sens fondamentalement véhiculé par cette racine. Un passage du rituel d'Anniwiyanni (VBoT 24 ii 2-5) semble témoigner de ce sens d'arha happešnai- (Bawanypeck 2005: 56-57) : n-ašta MÁŚ.GAL ANA ${ }^{\mathrm{D}}$ LAMMA lūlimi šipanti namma-(a)n arha happešnanzi nu-(̌́) šan ANA NINDA.GUR ${ }_{4}$.RA šer kēz kēzziya $z i k k i z z i$ «Il/elle sacrifie un bouc au dieu tutélaire lülimi. Ensuite on le démembre et il/elle le place sur du pain ordinaire de part et d'autre. » L'extrait indique que l'action décrite par arha happešnai- permet de subdiviser le corps de l'animal puis de disposer les morceaux de viande ainsi obtenus dans différents endroits.

A. Ünal (Ünal 1985: 432) a dressé la liste des autres verbes utilisés, quoique plus rarement, pour faire allusion à l'acte de débitage de la viande sacrificielle.

\section{Quatrième étape : la cuisson de la viande}

Il a déjà été signalé que la viande sacrificielle pouvait être offerte crue à la divinité ${ }^{21}$. Elle peut, d'un autre côté, être cuite, action décrite par le verbe zanu- . L'on peut distinguer deux principales techniques de cuisson : 1) dans un pot ;2) au happina-/feu.

1) La cuisson « au pot» est le plus souvent désignée par ${ }^{\mathrm{DUG}}$ ÚTUL anda zanu- « cuire

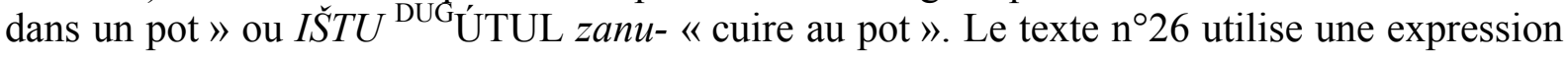

\footnotetext{
${ }^{20}$ Voir en particulier les mentions qui sont faites aux « neuf parties du corps » : HED III: 113.

${ }^{21}$ Voir le premier volet de cet article.
} 
différente, à savoir (UDU) UTÚL iya- «faire (le mouton) au pot». Le sumérogramme ${ }^{\mathrm{DUG}}$ ÚTUL équivaut à l'akkadien diqārum, ce dernier désignant un récipient à fond arrondi utilisé pour servir ou réchauffer les mets (Gelb et al. (éd.) 1959: 157-159). Les textes mésopotamiens indiquent que ce récipient est surtout utilisé pour chauffer ou servir les aliments liquides et notamment les bouillons de viande (Gelb et al. (éd.) 1959: 158). Dans le cas des sources hittites, certaines parties du corps de l'animal sacrifié sont jetées dans ce pot, sans doute pour en faire une sorte de ragoût ${ }^{22}$. Le texte $n^{\circ} 48$ atteste de l'existence d'un tel ragoût, qui semble dans ce contexte être agrémenté de gruau (akkadien arsānum).

2) L'autre type de cuisson est décrite par happinit zanu- " cuire au happina- » ce qui équivaut vraisemblablement à IZI-it/pahhuenit zanu- "cuire au feu ». C'est d'ailleurs ce qu'indique le texte $\mathrm{n}^{\circ} 30$ qui, dans ses différentes versions parallèles, fait alterner happinit (écrit fautivement happišnit) et IZI-it. A la lumière de cette équivalence, A. Goetze (Goetze 1970) traduisait happinit zanu- par « cook over a flame». Cette traduction a été suivie par la majorité des hittitologues qui se sont penchés sur la question. A. Ünal (Ünal 1985: 435), par exemple, traduit cette même expression par «auf der offenen Flamme rösten ». J. Puhvel (HED III: 121) suggère quant à lui de traduire plus spécifiquement happina- par «baking kiln, fire-pit, broiler (oven) ». Il y a en effet tout lieu de penser que cette technique de cuisson consiste à faire rôtir les viandes en les introduisant dans un foyer. Les textes ne nous permettent pas de préciser si ce foyer est fixe ou portatif. Seul le texte $n^{\circ} 44$ décrivant une fête célébrée par un prince témoigne peut-être de la manière dont les viandes étaient disposées dans ou au-dessus du happina-. On y mentionne en effet les šuppa «embrochés » (išgaranta) sur un bâton, ce qui pourrait être la technique utilisée pour faire rôtir ces viandes au happina/feu, à l'inverse de la « technique du pot». Par ailleurs, le texte $\mathrm{n}^{\circ} 1$ fait allusion à l'utilisation d'un foyer en bronze (GUNNI ZABAR) pour " apprêter» le foie et le cœur des victimes. Etant donné que, d'après les textes, ces deux organes sont presque toujours rôtis au happina/feu (voir tableau ci-dessous), et ceci quelle que soit la région d'origine du rituel décrit, il n'est pas impossible que le sumérogramme GUNNI soit employé dans le texte $n^{\circ} 1$ pour le hittite happina-. Si tel était bien le cas, cela signifierait que le happina- est un foyer portatif qui peut être en partie constitué de bronze. Cela reste toutefois une simple hypothèse, les textes ne permettant pas d'affirmer cette équation happina- $=$ GUNNI.

Il n'est pas rare que les deux formes de cuisson se côtoient dans les rituels hittites. Il semble que le choix de l'une ou de l'autre se fasse principalement en fonction de la nature des parties anatomiques de l'animal. Le tableau suivant tente de mettre en évidence la répartition des deux modes de cuisson dans la soixantaine de textes qui sont étudiés dans cet article en plusieurs volets. Les nombres qui sont reportés dans chaque case correspondent aux nombres d'occurrences de telle ou telle partie anatomique animale. Seuls les passages dénués de lacune ont été pris en compte.

\begin{tabular}{|c|c|c|}
\hline partie anatomique & ${ }^{\text {DUG ÚTUL }}$ & happina-/feu \\
\hline tête & 5 & 0 \\
\hline oreille & 0 & 1 \\
\hline épaule & 1 & 2 \\
\hline cœur & 0 & 23 \\
\hline patte antérieure $(«$ main ») & 3 & 0 \\
\hline poitrine & 6 & 0 \\
\hline
\end{tabular}

\footnotetext{
${ }^{22}$ Mode de cuisson également attesté en Syrie à l'époque de Mari (ARMT 21) et à l'époque néo-babylonienne (Joannès 2000: 335).
} 


\begin{tabular}{|c|c|c|}
\hline jarret $^{\text {' }}$ (KURIDU) & 2 & 0 \\
\hline côtelettes $^{\text {poumon }}$ & 4 & 1 (c. courtes) \\
\hline foie & 1 & 0 \\
\hline gigot $^{\text {? }}$ & 1 & 26 \\
\hline patte postérieure (« pied ») & 7 & 0 \\
\hline $\begin{array}{c}\text { haut des pattes antérieures et } \\
\text { postérieures }(\text { kudur })\end{array}$ & 3 & 0 \\
\hline muh(ha)rai- & 2 & 0 \\
\hline auli- & 1 & 0 \\
\hline «os pur » (parkui haštai) & 1 & 0 \\
\hline
\end{tabular}

Les principales observations que l'on peut émettre à la suite de l'étude de ce tableau sont les suivantes :

- Le statut particulier du foie et du cœur semble manifeste, tout d'abord parce qu'ils sont cuits de manière différente des autres parties anatomiques (ils sont systématiquement cuits au happina-, à une exception près à savoir le texte $n^{\circ} 29$ ) mais aussi par leur omniprésence dans les textes. Il faut toutefois préciser que l'écart numéraire mis en évidence par ce tableau entre foie-cœur et les autres parties anatomiques est partiellement comblé par les occurrences des viandes crues. De nombreux textes témoignent en effet d'une sorte d'opposition entre foie et cœur cuits au happina- et les autres viandes qui restent crues.

- Une partie anatomique particulière est presque toujours cuite de la même manière, même s'il existe deux exceptions notables: 1) les côtelettes courtes sont traitées différemment des côtelettes normales ; 2) l'épaule est tantôt cuite au pot, tantôt rôtie au happina-.

La coexistence de deux modes de cuisson pour les viandes sacrificielles (dans un pot pour faire bouillir certains morceaux et dans un foyer pour faire rôtir les autres) est un élément bien attesté dans la Grèce ancienne, comme en témoigne notamment le mythe de Lycaon que j'ai eu l'occasion d'évoquer dans un autre article ${ }^{23}$.

Il est rare que les textes fassent allusion au lieu dans lequel la cuisson de la viande sacrificielle se déroule. Les textes $n^{\circ} 4$ et 49 témoignent toutefois du fait que cette action peut être effectuée dans l'enceinte du temple même.

Cinquième étape : manipulation et dépôt de la viande devant la divinité

Que l'on ait cuit la viande ou non, le geste le plus important du sacrifice est vraisemblablement le dépôt de la viande consacrée devant la divinité, puisque c'est lui qui, en dehors de la dédicace de la victime par le prêtre ou le commanditaire du rituel, exprime le plus clairement l'offrande. Il arrive que l'action du dépôt de ce qui va constituer le repas divin soit menée tout de suite après la préparation de la viande (textes $\mathrm{n}^{\circ} 14,57$, etc.). Le foie et le cœur sont le plus souvent déposés sur du pain, et non directement sur l'emplacement sacrificiel (textes $n^{\circ} 8,44$, etc.). La nature de ce dernier peut être la suivante :

\footnotetext{
${ }^{23}$ Voir Mouton 2004: 308.
} 
- $\quad$ sur ou face à une table elle-même déposée devant l'image de la divinité (textes $\mathrm{n}^{\circ} 5$, 14,15 , etc.) ; cette table est parfois qualifiée de «table du sacrifice » (texte ${ }^{\circ} 14: \check{S} A$ SÍSKUR ${ }^{\text {GIS }}$ BANŠUR) ou d'autel (texte $n^{\circ} 4$ : ZAG.GAR.RA).

- devant le trône de la divinité (texte $\mathrm{n}^{\circ} 51$ ).

- directement par terre devant la divinité (texte $\mathrm{n}^{\circ} 22$ ).

- $\quad$ sur du feuillage qui a été étalé par terre au préalable (texte ${ }^{\circ} 60$ ).

- au-dessus d'un puits funéraire dans le cas d'un rituel d'évocation (texte $n^{\circ} 18$ ).

- dans un dépôt (ÉSAG) dans le cas d'un rituel analogue à celui de Tunnawiya (texte $\left.\mathrm{n}^{\circ} 35\right)$.

- dans un panier ( ${ }^{\mathrm{GIS}}{ }^{\text {irh }}$ ūiti) pendant la fête hišuwa (texte ${ }^{\circ} 20$ ).

Les viandes sont presque toujours déposées devant une image de la divinité concernée par la cérémonie religieuse. Cette image est soit une statue ${ }^{24}$ soit une stèle huwaši- (textes $\mathrm{n}^{\circ} 4,27$ ).

Dans d'autres circonstances, le dépôt de la viande sacrificielle est précédé par une série d'actions dont la fonction n'est pas toujours très claire. Il a déjà été fait allusion à la possibilité de pratiquer une interrogation hépatoscopique sur le foie de l'animal (texte $\mathrm{n}^{\circ} 15$ ) mais bien d'autres manipulations sont mentionnées dans les textes. Dans les rituels et fêtes religieuses kizzuwatniens par exemple, il n'est pas rare qu'une portion de la viande sacrificielle soit prélevée pour être plongée dans l'huile puis placée dans un récipient huprušhi- (textes $\left.\mathrm{n}^{\circ} 17,23,64\right)$ ou ahrušhi- (textes $\left.\mathrm{n}^{\circ} 17,20\right)$. Les textes $\mathrm{n}^{\circ} 17$ et 47 indiquent que les deux récipients peuvent de temps à autre être employés au cours d'un même rituel. A côté de cela, plusieurs gestes peuvent être effectués par le commanditaire du rituel, ceci vraisemblablement dans le but de l'impliquer davantage dans le sacrifice. Ainsi, le commanditaire du rituel peut se voir attribuer un couteau avec lequel il lui faudra couper une partie anatomique de $1^{\prime}$ animal (texte $\mathrm{n}^{\circ} 17:$ le foie et le cœur ; texte $\mathrm{n}^{\circ} 53$ : le gigot ${ }^{?}$ et $l^{\prime} e k d u$-). Parfois, le commanditaire du rituel ne coupera pas vraiment le corps de la victime, mais se contentera de tenir le couteau près d'une partie anatomique précise, peut-être pour signifier qu'il est à l'origine du sacrifice (texte $\mathrm{n}^{\circ} 62$ ). Un autre geste consiste pour le commanditaire du rituel à déchirer un morceau de viande avec les dents (texte ${ }^{\circ} 53$ ).

Les textes ne disent rien sur la façon dont les dieux se nourrissent des offrandes qu'on leur fait. Il semble que le dépôt de la viande devant leur effigie suffise à remplir cet office ${ }^{25}$. Il s'agit donc bien d'un acte symbolique qui se fait sans avoir recours à l'immolation par le feu de la victime, contrairement à ce que nous savons des pratiques de la Grèce ancienne notamment. Le fait que le repas des dieux soit conçu comme un phénomène purement symbolique justifie déjà la consommation ponctuelle par les mortels des viandes consacrées.

\section{Sixième étape : consommation de la viande par les participants de la cérémonie}

Contrairement à ce que l'on a pu déduire de certains textes hittites bien connus, comme celui des instructions pour le personnel du temple, dans lequel il est question d'un tabou placé sur les aliments divins ${ }^{26}$, il me semble que les viandes consacrées ne sont pas toujours prohibées à la consommation humaine. On a pu penser que seules les viandes qui n'ont pas été offertes aux dieux sont mangées par les mortels (de Martino 2004: 350). Cependant, les textes appellent ces dernières šuppa, à l'instar des morceaux qui sont présentés sur l'autel divin. Il faut donc en déduire qu'elles étaient elles aussi consacrées. Ainsi, plusieurs extraits étudiés ici

\footnotetext{
${ }^{24}$ Même si les textes ne se donnent pas la peine de le préciser, il paraît évident que la divinité est le plus souvent représentée par une statue.

${ }^{25}$ Sur le même sujet dans le contexte mésopotamien, voir Oppenheim 1964: 192.

${ }^{26}$ Voir en dernier lieu de Martino 2004: 349.
} 
mentionnent expressément la consommation humaine de šuppa (textes $\mathrm{n}^{\circ} 24,41,45$ ). Cela reviendrait à dire que c'est généralement l'animal tout entier qui est consacré à la divinité, mais que les différentes parties de son corps sont par la suite partagées entre ce dieu et les hommes après l'accomplissement du sacrifice. Il faudrait donc nuancer ce qui avait été formulé par B. J. Collins et S. de Martino : étant donné que c'est le corps entier de l'animal qui est offert à la divinité, toutes les viandes qui en sont extraites doivent être considérées comme sacrées, y compris celles qui ne sont pas déposées sur l'autel. Mais cette sacralité n'interdit pas aux hommes de consommer une partie de la viande sacrificielle. Par ailleurs, le texte $\mathrm{n}^{\circ} 45$ (et peut-être également le passage lacunaire $\mathrm{n}^{\circ} 41$ ?) témoigne de la consommation des šuppa qui avaient été déposées devant la divinité. Un phénomène similaire a été observé par F. Joannès dans les sources néo-babyloniennes, qui nous offrent de plus amples renseignements sur ce point précis (Joannès 2000: 335) : "Les animaux sont engraissés dans les étables du temple, puis mis à mort et découpés. Une partie de la viande n'était probablement pas consommée par la divinité et ce qui était servi sur la table d'offrande était ensuite récupéré par le sanctuaire. Globalement, toute la viande fournie par un animal était réattribuée à un certain nombre de gens membres du personnel du temple, au prorata de la fonction qu'ils occupaient dans le sanctuaire. Une part non négligeable était attribuée au roi, en tant que l'un des principaux fournisseurs des ressources du temple. [...] On peut donc établir que cette redistribution des repas servis aux dieux affectait deux sortes de viandes : la première sous forme brute, mise de côté au moment du découpage de l'animal, la seconde après préparation culinaire, redistribuée sous forme de plat cuisiné. »

On doit donc supposer que le tabou pesant sur la nourriture divine dont se fait écho non seulement les instructions au personnel du temple mais également la prière de Kantuzzili (Lebrun 1980: 112 et 116) ne concerne pas tous les šuppa de nos cérémonies religieuses, ce dernier terme étant employé pour tous les morceaux de viande issus d'un animal consacré, qu'ils soient offerts ou non aux dieux ${ }^{27}$. D'un autre côté, le rituel kizzuwatnien KBo 8.86 Ro 13'-14' indique qu'un animal peut parfois être réservé aux dieux et donc strictement interdit à la consommation humaine (Haas/Wilhelm 1974: 262-263) : $n u k \bar{e} \mathrm{MUŠEN}^{\mathrm{HÁ}}$ SILA $_{4}{ }^{\mathrm{HÁ}} 1$ AMAR-y[a $\ldots]$ šuppaéš $\bar{U} L-a \check{s}$ kuiški ézzazi «Ces oiseaux, agneaux et un veau [...] sont sacrés. Personne ne les mange. »

A côté de ce témoignage qui va dans le sens de ceux déjà mentionnés auparavant et qui corrobore l'existence d'un tabou alimentaire sur certaines viandes sacrificielles, d'autres textes indiquent au contraire que les parties animales qui semblent les plus sacrées peuvent, dans des contextes précis, être consommées par les hommes. C'est particulièrement le cas du foie dans le texte $n^{\circ} 41$. Ces apparentes contradictions dans les témoignages textuels illustrent bien, à mon sens, l'existence de traditions sacrificielles locales au sein de l'Anatolie hittite ${ }^{28}$.

Le texte $n^{\circ} 58$ relatif au culte de Huwaššanna témoigne d'un mode particulier de consommation des viandes consacrées. On y emploie en effet l'expression šuppa IŠTU GEŠTIN $a k u$ - «boire les šuppa avec du vin ». Le CHD a sans doute raison de suggérer que derrière cette expression se cache une pratique consistant à mélanger au vin de petits morceaux de viande ${ }^{29}$. Quoi qu'il en soit ce témoignage doit également être versé au dossier des šuppa consommées par les hommes.

Même quand une partie de la viande consacrée peut être consommée par les mortels, tous les participants ne sont sans doute pas autorisés à prendre part au festin. On sait en effet que, de

\footnotetext{
${ }^{27}$ Je remercie le Dr. Y. Cohen qui a gentiment discuté ce thème précis avec moi.

${ }^{28}$ Sur ce thème, voir ma contribution au VIème Congrès International d'Hittitologie déjà mentionné ci-dessus.

${ }^{29} \mathrm{CHD}$ šuppa, à paraître.
} 
manière plus générale, les banquets cultuels n'étaient pas accessibles à tous (Collins 1995: 8689).

Annexe : extraits de textes ${ }^{30}$

\section{$\underline{n^{\circ} 56}$}

KUB 25.23 bord gauche 3-5 = inventaire de temples (éd. Carter 1962: 163 et 173-174). Origine incertaine. Tudh. IV.

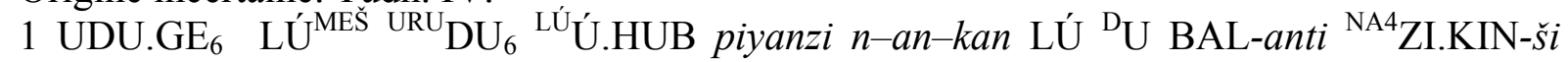
hūkanzi šuppa huešauwaz zēantaz tiyanzi « Les hommes du Tell du Sourd donnent un mouton noir et l'homme du dieu de l'orage le sacrifie. On (l')abat près de sa (= le dieu de l'orage) stèle et on place les šuppa crus (et) cuits. »

\section{$\mathbf{n}^{\circ} \mathbf{5 7}$}

KUB 60.121 obv. 2'-9' = rituel de l'AZU (éd. Popko 1991: 239-241). Origine kizzuwatnienne. MH/MS.

x x [... UDU a]nda ūnniyanzi $n-a n-k a n{ }^{\mathrm{LU}} \mathrm{AZU} k \bar{e} d a<\check{S}^{>}>A N A 3 \mathrm{DINGIR}^{\mathrm{MEŠ}}$ šipanti n-ankan parā penniyanzi n-an hattanzi n-ašta UZU šuppa huišu ${ }^{\mathrm{UZU}} \mathrm{GABA}{ }^{\mathrm{UZU}} \mathrm{ZAG} . \mathrm{LU}$ SAG.DU ${ }^{\mathrm{MES}}$ GİR $^{\mathrm{MES}}$ KUŠ.UDU danzi n-at PANI GIŠ BANŠUR AD.KID tianzi šer arha-ma$(\check{s}) \check{s} a n{ }^{\mathrm{UZU}} \mathrm{I} . \mathrm{UDU}$ huittiyanzi $\S \mathrm{UZU}^{\mathrm{U}} \mathrm{NÍG}$.GIG-ma UZU Š̀̀ IZI-it zanuanzi mahhan-ma-at zeyari n-at-kan anda udanzi nu ${ }^{\mathrm{LU}} \mathrm{AZU} 3$ NINDA.SIG kédaš DINGIR ${ }^{\mathrm{MES}}$-aš paršiya šer-a$(\check{s})$ šan ${ }^{\mathrm{UZU}} \mathrm{NÍG} . \mathrm{GIG}{ }^{\mathrm{UZU}} \breve{\mathrm{S} A ̀ ~} d \bar{a} i n$-at PANI DINGIR ${ }^{\mathrm{MEŚ}} d \bar{a} i$ «On amène [... mouton]. L'exorciste le sacrifie à ces trois divinités et on l'emmène. On le tue. On prend (ses) šuppa crus, (à savoir) la poitrine, l'épaule, la tête ${ }^{31}$, les « pieds » et le cuir et on les place devant une table de roseau. Mais on en retire la graisse. § On fait cuire le foie (et) le cœur au feu. Quand ils sont cuits, on les apporte. L'exorciste rompt trois pains plats pour ces divinités et pose le foie (et) le cœur par dessus. (Puis) il les place face aux divinités. »

\section{$\underline{\mathbf{n}^{\circ} 58}$}

$\overline{\text { KUB }} 27.65$ i $9=$ culte de Huwaššanna. Origine louvite. NH.

[EGIR-a]nda-ma šuppa 7-ら̌U IŠTU GAL GEŠTIN akuw[anzi] « [Ensui]te on bo[it] sept fois les šuppa avec une coupe (de) vin. »

\section{$\underline{n^{\circ} 59}$}

KUB 17.35 ii 30-31 = inventaire de temples (éd. Carter 1962: 128 et 141). Origine incertaine. Tudh. IV.

lukatti-ma $\mathrm{U}_{4} \cdot \mathrm{KAM}^{\mathrm{UZU}}{ }^{\mathrm{NÍG}}$ GIG šuppa UZU ${ }^{\mathrm{TU}}{ }^{\text {šiyami DÙ-zi PANI DINGIR }}{ }^{\text {LIM }}$ tianzi « Le lendemain (est) le jour (du) foie. Il/elle fait (des) šuppa une bouillie šiyami à la viande (et) on (la) place face à la divinité. »

\section{$\underline{\mathbf{n}^{\circ} \mathbf{6 0}}$}

KUB 9.32 obv. 19-21 ; rev. 24-31 // KUB 9.31 iii 36-39; iv 33-40, HT 1 iii 29-32 ; iv 36-43, KUB 41.18 iii 9-12 = rituel d'Ašhella de Hapalla (Arzawa) contre une épidémie (éd. Dinçol 1985: 1-40). NH.

\footnotetext{
${ }^{30}$ La numérotation des extraits est en continuation avec celle utilisée dans les premiers volets de cet article. J'invite par conséquent le lecteur à se référer à ces deux précédentes publications pour prendre connaissance des textes étudiés auparavant.

${ }^{31}$ Le pluriel est sans doute une faute d'étourderie du scribe.
} 
kuiš-wa DINGIR ${ }^{L I M} k \bar{\imath} \mathrm{UG}_{6}$-an (var. $\mathrm{UG}_{6}-k a n$ ) iyat kinun-a-wa kāša UDU. ̌̌s ${ }^{\mathrm{HÁ}}$ arantari $n u$-war-at IŠTU ${ }^{\mathrm{UZU}} \mathrm{NÍG.GIG}{ }^{\mathrm{UZU}} \breve{\mathrm{S}} \mathrm{A} \dot{U}{ }^{\mathrm{UZU}}{ }^{\mathrm{U}} \mathrm{R}$ (var. UZU-ya; ${ }^{\mathrm{UZU}} \mathrm{I}-y a$ ) mekki warganteš / rev. 24-32: nu-kan GU $_{4} A N A{ }^{\mathrm{D}} \mathrm{IM}$ šipanti ${ }^{32}$ UDU.SÍG+MUNUS-ma-kan ANA ${ }^{\mathrm{D} U T U}$ šipanti 3 UDU-ma-kan (var. UDU.NITA) humandaš DINGIR ${ }^{\mathrm{MEŠ}}$-aš šipandanzi $n u{ }^{\mathrm{GIS}}$ lahhurnuzzi dagān išparranzi nu ${ }^{\mathrm{UZU}} \mathrm{GABA}{ }^{\mathrm{UZU}} \mathrm{ZAG}$.LU ZAG-an UZU NÍG.GIG ${ }^{\mathrm{UZU}} \mathrm{S} \breve{\mathrm{A}}{ }^{\mathrm{GIS}}$ lahurnuziaš $d \bar{a} i^{33}$ NINDA.GUR 4 .RA-ya ziyandaz EGIR-pa dāi $n u{ }^{\mathrm{D}} \mathrm{UTU} \breve{S} A M \bar{E}{ }^{\mathrm{D}} \mathrm{IM}^{\mathrm{SIN}} \mathrm{DINIR}^{\mathrm{MES}}{ }_{-y a}$ hūmanteš 3- ̌́ ekuzi (var. akuwanzi) nu-za adanzi n-at-za arha uwanzi «(Toi), la divinité qui a provoqué cette épidémie, des béliers se tiennent (ici) en ce moment ! Ils (sont) bien gras avec leur foie, leur cœur et leurs membres (var. la viande ; la graisse). / rev. 24-32 : Il/elle sacrifie un bœuf au dieu de l'orage, il/elle sacrifie une brebis au dieu Soleil et on sacrifie trois moutons (var. moutons mâles) à tous les dieux. On étale du feuillage par terre et il/elle place la poitrine, l'épaule droite, le foie (et) le cœur sur les feuilles. Il/elle repose le pain ordinaire cuit. Il/elle boit (var. on boit) à trois reprises le dieu Soleil du ciel, le dieu de l'orage et tous les dieux. On mange et on s'en va. »

\section{$\underline{n^{\circ} 61}$}

KBo 29.72 + KBo 14.96 Ro 7'-9' ; 13'-14' = fête de Huwaššanna witaššiaš (éd. Kühne 1986: 85-86). Origine louvite. MS.

BEL SÍSKUR-ma-kan 1 UDU ${ }^{\mathrm{D}}$ huwaššanna [š]ipanti nu-za mahhan ${ }^{\text {LÚMUHALDIM UDU }}$ hūganna êpzi [U]ZU aulišs šiēzzi / Ro 13'-14': § BEL SÍSKUR-ma-kan 1 UDU AN[A] ${ }^{\mathrm{DUTU}}$

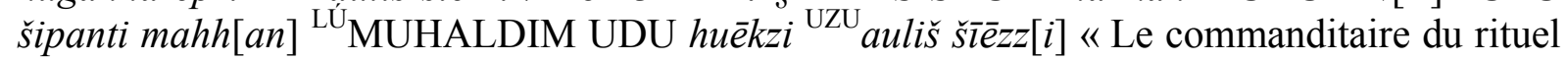
sacrifie un mouton de Huwaššanna. Quand le cuisinier se met à abattre le mouton, (le sang) de l'auli coule. / Ro 13'-14': § Le commanditaire du rituel sacrifie un mouton pour le dieu Soleil. Quand le cuisinier abat le mouton, (le sang) de l'auli coule. »

\section{$\underline{n^{\circ} 62}$}

KBo 15.33 + KBo 15.35 iii 9-28 = fête pour le dieu de l'orage de Kuliwišna (éd. Glocker 1997: 70-71). Origine hatto-hittite. MH/MS.

$n-a \grave{s}-k a n{ }^{\mathrm{LU}}{ }_{\mathrm{EN}} \dot{E}^{T I M} A N A{ }^{\mathrm{D}} \mathrm{IM}^{\mathrm{URU}}$ kuliuišna šipanti nu UDU.ŠIR GU $4 . \mathrm{MAH}-y a$

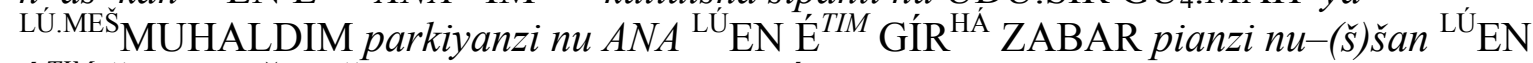
É $^{T I M} \check{S} A$ UDU.ŠIR $\breve{S} A$ GU 4 .MAH-ya auliya GÍR ZABAR-it QATAM dāi $\S$ LÚ.MEŠ MUHALDIM-ma-aš ištanāni hukanzi nu kuiš LÚ MUHALDIM huikuanzi tarahzi nu-

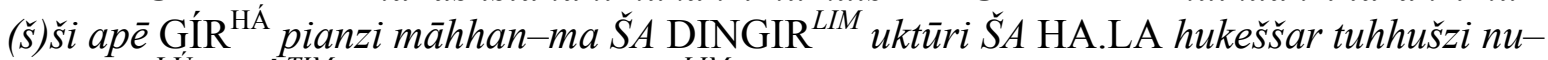
zan mān ${ }^{\mathrm{LU}} \mathrm{EN} \mathrm{E}^{T I M}$ kuitki ANA DINGIR ${ }^{L I M}$ maltan harzi mān UNUTUM kuitki mān $\mathrm{GU}_{4}$ UDU $\S n u-(\check{s})$ šan UNUT INA NINDA.ERÍN.MEŠ tianzi $\mathrm{GU}_{4}-m a$ UDU ${ }^{\text {LÚ MUHALDIM }}$ tuhhuiš[nit] šuppiyahhi n-uš-kan anda ūnnianzi nu-šmaš ${ }^{\mathrm{LU}} \mathrm{EN} \dot{\mathrm{E}}^{T I M} \mathrm{EGIR}-\check{S} U N U U \check{S} G E N$

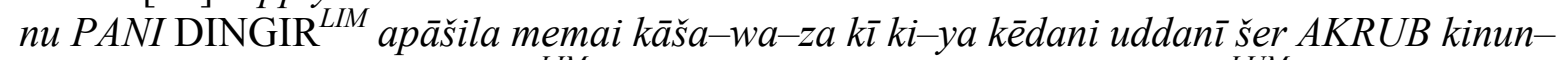
$a-w a r-a[t] k \bar{a} s ̌ a$ ANA DINGIR ${ }^{L I M}$ udahhun nu-war-at-za-kan DINGIR ${ }^{L U M}$ menahhan $[$ da $\bar{u}$ ški] « Le maître de maison fait un sacrifice au dieu de l'orage de Kuliwišna. Les cuisiniers soulèvent un bélier et un taureau ${ }^{34}$. Ils donnent des couteaux de bronze au maître de maison.

\footnotetext{
${ }^{32}$ Sur KUB 9.32 rev. 24, on lit ši-pa-an-da-an-ti. Les signes DA et TI ont été inscrits sur une érasure. Le signe TI semble avoir remplacé un signe ZI dont il reste les deux derniers Winkelhaken. Il paraît donc clair que le scribe a voulu transformer une troisième personne du pluriel šipandanzi en troisième personne du singulier šipanti. Les deux duplicats KUB 9.31 iv 33 et HT 1 iv 20' donnent tous les deux une troisième personne du singulier. KUB 9.31 change de paragraphe après šipanti.

${ }^{33 \mathrm{GIS}}$ lahurnuziaš dāi a été omis dans KUB 9.31 et HT 1.

${ }^{34}$ Peut-être faut-il suivre la suggestion du CHD P: 156 qui traduit : « and the cooks lift (the heads of) the (living) ram and the bull (and they give bronze knives to the master of the house, who places his hand with the bronze knife on the jugular vein of the ram and the bull ». Il faut noter que la traduction pourtant judicieuse de auli- par « jugular vein » ne s'accorde pas très bien avec les quelques autres attestations de ce terme (voir la bibliographie dans Anatomie animale II).
} 
Le maître de maison pose la main sur l'auli du bélier et du taureau (muni) d'un couteau de bronze. $\S$ Les cuisiniers les abattent sur l'autel. Le cuisinier qui peut procéder à l'abattage, on lui donne lesdits couteaux. Quand l'abattage des parts (de viande) habituelles de la divinité est fini, si le maître de maison a voué quelque chose à la divinité, que ce soit un objet quelconque, que ce soit un bœuf (ou) un mouton, $\S$ on dépose l'objet sur (m. à m. « dans ») un pain de soldats. Mais le cuisinier consacre (l'éventuel) bœuf (ou) mouton avec du tuhhueššar. On les amène, le maître de maison s'agenouille derrière eux et dit en face du dieu en personne : « Je viens de (te) vouer celui-ci et celui-là pour cette affaire. Maintenant, je viens de les apporter à la divinité. [Vois]-les, (eux qui sont) devant (toi), divinité ! »

\section{$\underline{n^{\circ} 63}$}

KUB 57.84 iii 2'-4' = liste d'offrandes pour des cultes locaux (éd. Forlanini 1984: 256-257). Origine hatto-hittite. NS.

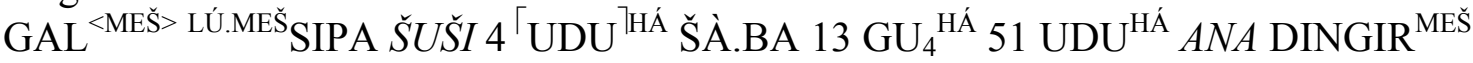
hukanzi « Les chefs des bergers abattent soixante-quatre moutons dont treize bœufs et cinquante-et-un moutons pour les dieux. »

\section{$\underline{n^{\circ} 64}$}

KBo 33.204 iv 7'-12' = liste de divinités hourrites (transcr. Wegner 2002: 79-81). Origine kizzuwatnienne. NS.

[(n-ašta GU4.MAH (var. $\left.{ }^{\mathrm{GU} 4} \mathrm{ÁB}\right)$ anda) pi]nniyanzi (var. ūnni[yanzi]) [(nu-šši-kan ANA SA)]G.DU- $\breve{S} U$ (var. $\left.n u-(\check{s}) \check{s} a n{ }^{\mathrm{LU}} \mathrm{SANGA} A N A{ }^{\mathrm{GU} 4}[\mathrm{AB}]\right){ }^{\mathrm{UZU}} \mathrm{ZAG} . \mathrm{LU}-a \check{s}-(\check{s}) a\left[\left({ }^{\mathrm{G}}{ }^{\mathrm{S}} \mathrm{ERIN} d \bar{a} i\right.\right.$ $n$-an-kan ui)]tenit šipanti [(n-ašta anāhi dāi n)]-at-kan (var. n-at-šan) huprušhiya [(piššiyazzi ${ }^{35} n$-ašta) GU 4 .MA]H IŠTU KAŠ.GEŠTIN (var. GEŠTIN) [(ANA ${ }^{\mathrm{D}}$ hepat šipa)]nti « On [a]mène un taureau (var. une vache). Il (var. le prêtre) place du bois de cèdre (sur) sa ${ }^{36}$ tête et ses épaules. Il le sacrifie avec de l'eau. Il prend un morceau (de viande) et le jette dans un récipient huprushi-. Il sacrifie le taureau avec du vin (de) bière (var. du vin) pour Hepat. »

\section{$\underline{n^{\circ} 65}$}

KUB 56.49 Ro 6'-9' // KUB 56.48 ii 3-6 (NS) et KBo 2.4 iii 1-3 (NH) = fête du mois (éd. de KBo 2.4 : Haas 1970: 284-285). Origine hatto-hittite avec apports kizzuwatniens. NH.

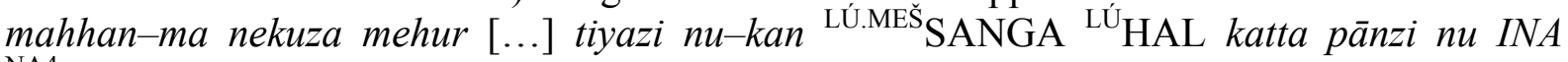

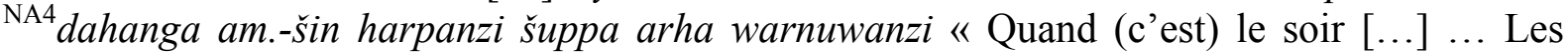
prêtres (et) l'haruspice descendent. Ils assemblent l'offrande ambašši dans le dahanga, ils font brûler les šuppa. »

\section{Bibliographie $^{37}$}

Bawanypeck, D.

2005 Die Rituale der Auguren, THeth 25, Heidelberg.

Beckman, G.

à paraître "Blood in Hittite Ritual”, Gedenkschrift Neu.

\footnotetext{
${ }^{35}$ Fin du paragraphe dans KBo 22.180 i 5.

${ }^{36}$ Le possessif renvoie à l'animal, comme le prouve la var. ANA ÁB.

${ }^{37}$ Le lecteur pourra retrouver les abréviations utilisées dans cet article dans : H. G. Güterbock/H. A. Hoffner (éd.), The Hittite Dictionary of the Oriental Institute of the University of Chicago (= CHD), L-N, Chicago 1989:xxi-xxix; CHD P, Chicago 1997:vii-xxvi; CHD Š, Chicago 2002:vi-viii.
} 
Briquel, D.

2004

"Remarques sur le sacrifice étrusque", La fête. La rencontre des dieux et des hommes. Actes du $2^{e}$ colloque international de Paris organisé par les Cahiers KUBABA (6-7 décembre 2002), Paris: 133-153.

Carter, C. W.

1962

Hittite Cult-Inventories, PhD Chicago.

Cohen, Y.

2002

Taboos and Prohibitions in Hittite Society. A Study of the Hittite Expression natta āra ('not permitted'), THeth 24, Heidelberg.

Collins, B. J.

1995

"Ritual Meals in the Hittite Cult", Ancient Magic and Ritual Power (éds.

Meyer/Mirecki), Leiden/New York:77-92.

de Martino, S.

2004 "Purità dei sacerdoti e dei luoghi di culto nell'Anatolia ittita", Or NS 73: 348362.

Dinçol, A.

1985 "Ašhella ritualı (CTH 394) ile Hititlerde salgın hastalıklara karşı yapılan majik işlemlere toplu bir bakış”, Belleten 49/193: 1-40.

Durand, J. -L.

1979

"Bêtes grecques. Propositions pour une typologie des corps à manger", $L a$ cuisine du sacrifice en pays grec (éds. Detienne/Vernant), Paris: 133-157.

Gelb, I. J. et al. (éd.)

1959 The Assyrian Dictionary of the Oriental Institute of the University of Chicago 3: D, Chicago.

Glocker, J.

1997 Das Ritual für den Wettergott von Kuliwišna. Textzeugnisse eines lokalen Kultfestes im Anatolien der Hethiterzeit, Eothen 6, Florence.

Goetze, A.

$1970 \quad$ "Hittite šipant-", JCS 23: 77-94.

Forlanini, M.

1984 "Die 'Götter von Zalpa'. Hethitische Götter und Städte am Schwarzen Meer", ZA 74: 245-266.

Haas, V.

1970 Der Kult von Nerik. Ein Beitrag zur hethitischen Religionsgeschichte, Studia Pohl 4, Rome.

Haas, V./Jakob-Rost, L.

1984 "Das Festritual des Gottes Telipinu in Hanhana und in Kašha", AoF 11: 10-91. 
Haas, V./Wilhelm, G.

$1974 \quad$ Hurritische und luwische Riten aus Kizzuwatna, AOATS 3, Neukirchen-Vluyn.

Hoffner, H. A.

"Akkadian šumma immeru texts and their hurro-hittite counterparts", The Tablet and the Scroll. Near Eastern Studies in Honor of William H. Hallo (éds. Cohen/Snell/Weisberg), Bethesda: 116-119.

Joannès, F. 2000

"Le découpage de la viande en Mésopotamie", Les animaux et les hommes dans le monde syro-mésopotamien aux époques historiques, Topoi Supplément 2: 333-345.

Kühne, C.

1986

"Hethitisch auli- und einige Aspekte altanatolischer Opferpraxis", $Z A$ 76: 85117.

Lafont, B. 1999

“Sacrifices et rituels à Mari et dans la Bible", $R A$ 93: 57-77.

Lebrun, R. 1980

Hymnes et prières hittites, Homo Religiosus 4, Louvain-la-Neuve.

1993

"Aspects particuliers du sacrifice dans le monde hittite", Ritual and Sacrifice in the Ancient Near East. Proceedings of the International Conference organized by the Katholieke Universiteit Leuven (éd. von Quaegebeur), Orientalia Lovaniensia Analecta 55: 225-233.

Mazoyer, $\mathrm{M}$.

2002

"Quelques aspects du sacrifice sanglant chez les Hittites", Rites et Célébrations, Cahiers KUBABA 4/2: 81-89.

Mouton, A.

2004

"Une épreuve pour différencier l'homme du dieu : le "texte des cannibales" hittite (KBo 3.60) et quelques rapprochements, ou comment reconnait-on un dieu hittite ?", AoF 31: 303-319.

2004a "Le rituel de Walkui (KBo 32.176) : quelques réflexions sur la déesse de la nuit et l'image du porc dans le monde hittite", $Z A$ 94: 85-105.

à paraître "Torche et encens dans les rituels magiques et thérapeutiques hittites et mésopotamiens", L'objet magique en Egypte ancienne (éds. Tardieu/Yoyotte).

Oppenheim, A. L.

1964 Ancient Mesopotamia. Portrait of a Dead Civilization, Chicago.

Popko, M.

1991

“Eine ‘schwarze Tafel’ aus Boğazköy (KUB 40.121)”, AoF 18: 239-245.

Ünal, A.

1985 "Beiträge zum Fleischverbrauch in der hethitischen Küche: Philologische Anmerkungen $\mathrm{zu}$ einer Untersuchung von A. von den Driesch und J. 
Boessneck über die Tierknochenreste aus Boğazköy-Hattuša", Or NS 54: 419438.

Wegner, I.

2002

Hurritische Opferlisten aus hethitischen Festbeschreibungen. Teil II : Texte für Teschub, Hebat und ihren Kreis, ChS I/3-2, Rome.

Terminé en Février 2007

alicemouton@hotmail.com 